\title{
Ein rheinisch-schweizerischer Jubilar
}

\author{
Vor 450 Jahren wurde Fabricius Hildanus in Hilden (Deutschland) geboren. Doch seine \\ wichtigsten Jahre verbrachte er in der Schweiz. Als Chirurgus - Arzt konnte er wegen \\ mangelnder humanistischer Bildung nicht werden - arbeitete er in vielen Schweizer \\ Städten und führte komplizierte Operationen durch, nicht selten mit Hilfe seiner Frau.
}

Wäre in der Schweiz des frühen 17. Jahrhunderts die Weisheit ein Allgemeinplatz gewesen, wonach «behind every great man, there has to be a great woman»-der Heilkundige hätte ihr aus vollem Herzen zugestimmt. Die Gattin war ihm über ein langes Chirurgenleben die wichtigste Mitarbeiterin, doch nie war sie so innovativ wie an jenem Tag anno 1624, als sie Medizingeschichte schrieb - um dennoch auch in dieser im Schatten des Gemahls zu stehen. Er vertraute das Geschehen seiner Feder an: «Einem Bauern aus dem Tal S. Mie, nahe bei dem Bieler See, Benedictus Barquin mit Namen, sprang, als er einen Stahl bei einem Krämer kaufte und ein Stück gegen das andere schlug, ein Stückchen in jenen Teil der Hornhaut, wo die Regenbogenhaut gesehen wird und haftete dort fest in der
Wir - es war der Tribut eines grossen Operateurs an seine Frau, seine in der Ausübung der Heilkunst gleichberechtigte Partnerin. Da er nicht nur das Messer, sondern auch die Feder geschickt zu führen verstand, hat der heutige Leser anhand 600 derartiger Krankengeschichten einen faszinierenden Einblick in die Mühen - und oft auch Erfolge - der (nichtakademischen) Medizin in einer schwierigen Zeit. Sein Name war Wilhelm Fabry von Hilden oder auch Fabricius Hildanus. In Hilden, unweit Düsseldorfs in Deutschland gelegen, wird Fabricius als der grosse Sohn der Stadt in diesem Jahr aus Anlass seines 450. Geburtstages ausgiebig gefeiert. Fabricius wurde dort am 25. Juni 1560 geboren, seine wichtigsten und schaffensreichsten Jahre verbrachte er jedoch in der Schweiz.

\section{«Während ich nämlich mit beiden Händen die Augenlider öffnete, näherte sie einen Magneten so weit dem Auge, wie der Kranke es ertragen konnte»}

\footnotetext{
* Alle Zitate stammen aus Fabry W. Ausgewählte Observationes Wilhelm Fabry's von Hilden. Übers. von Rom. Joh. Schaefer. Eingel. von Karl Sudhoff. Leipzig: Johann Ambrosius Barth; 1914.
}

Korrespondenz: Dr. med. Dr. phil. Ronald D. Gerste 14801 Soft Wind Drive USA Gaithersburg, Maryland 20878 rdgerste@aol.com
Membran, und zwar nicht ohne erheblichen Schmerz. Als die Angehörigen mehrere Tage hindurch ihm zu helfen vergeblich sich bemüht hatten und die Schmerzen wie auch die Entzündung ausserordentlich zunahmen, kam er am 5. März zu mir nach Bern. Ich habe ihm zunächst eine recht gute Lebensweise vorgeschrieben und seinen Körper gereinigt sowohl durch Arznei, als auch durch Aderlass (denn er war vollblütig), dann habe ich erst durch Instrumente einige Tage hintereinander versucht, ob ich etwa den Splitter könnte herausziehen, aber er war so klein, dass es nicht möglich war. Da hat denn meine Ehefrau ein sehr geeignetes Mittel sich ausgedacht. Während ich nämlich mit beiden Händen die Augenlider öffnete, näherte sie einen Magneten so weit dem Auge, wie der Kranke es ertragen konnte. Als wir diesen nun einige Male zurückzogen, ist das Stahlstückchen endlich aus dem Auge an den Stein gesprungen, während wir es alle sehen konnten. Nachher ist der Kranke, als wir ein schmerzstillendes Augenwasser angewandt hatten, in kurzer Zeit gesund geworden.»*
Auf Wunsch des Vaters, eines Gerichtsschreibers, wurde der Knabe in die grosse Stadt Köln geschickt und besuchte dort wahrscheinlich ein Gymnasium, wo er mit der Gelehrtensprache Latein in Berührung kam. Doch schon 1569 starb der Vater, und ein anderer Bildungsweg musste eingeschlagen werden, auch weil das Rheinland von den Verheerungen des niederländischen Unabhängigkeitskrieges heimgesucht wurde. Doch ehe der Jüngling nach einer neuen Lehrstelle Ausschau halten konnte, schlug die grosse Seuche jener Epoche auch in Hilden zu: die Pest. Er erkrankte an dieser Epidemie und entging nur knapp einem frühen Ende. Möglicherweise hat diese eigene Erfahrung als Leidender ihn in dem Vorhaben unterstützt, sich der Heilung von Krankheiten zu widmen. Arzt konnte er mangels der erforderlichen humanistischen Ausbildung nicht werden, doch der handwerkliche Beruf des weniger angesehenen Chirurgen, auch Bader oder Wundarzt genannt, stand ihm offen.

Fabricius absolvierte eine Lehre zum Chirurgus bei einem Wundarzt in Neuss und dann bei Cosmas 
Slotanus, dem Hofchirurgen im Schloss zu Düsseldorf. Slotanus hatte beträchtlichen Einfluss auf die Denkweise des jungen Adlatus. Der Meister trichterte dem Lehrling förmlich ein, dass nur eine genaue Kenntnis der Anatomie des menschlichen Körpers einen Chirurgen zu verantwortungsvollem Handeln befähigt. Gelernt hatte Slotanus diese Maxime bei dem Begründer der wissenschaftlichen Anatomie der Neuzeit, bei Andreas Vesalius, dessen grandioses Werk De humani corporis fabrica 17 Jahre vor Fabricius' Geburt erschienen war und in dessen 639 Seiten dieser sich beim Studium von Bau und Funktion der sterblichen Hülle des Menschen zweifellos vertiefte. Im Düsseldorfer Schloss scheint es die strikte Standestrennung von

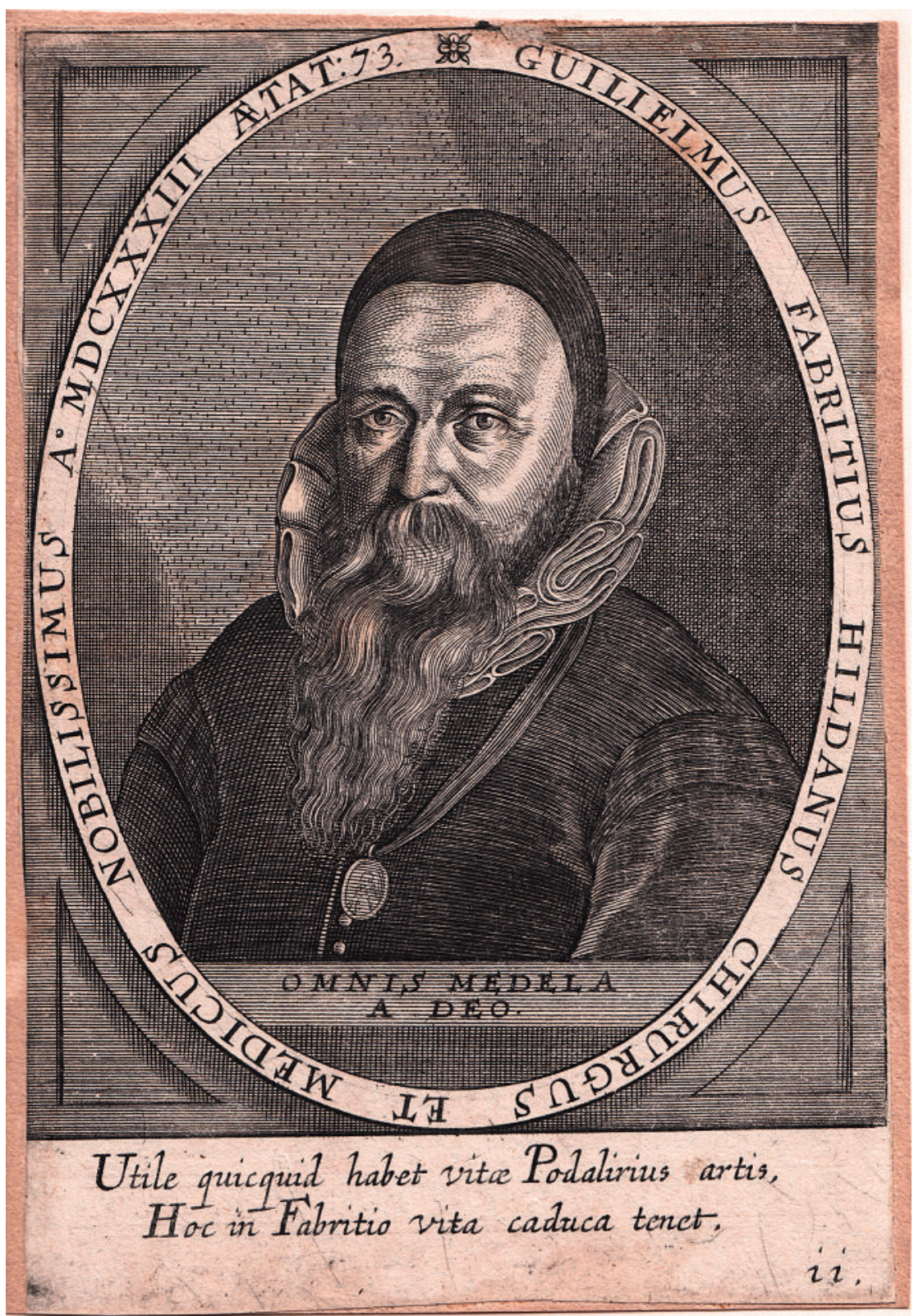

Der 73-jährige Wilhelm Fabry, genannt Fabricius Hildanus, damals Berner Stadtchirurg. Kupferstich aus: Jean-Jacques Boissard: Bibliotheca Chalcographica. Heidelberg 1669. Quelle: Medizinhistorisches Archiv der Universität Zürich, Sig. PS-a.
Chirurgen und akademisch gebildeten Ärzten sowie den damit oft verbundenen Hochmut der Letzteren nicht gegeben zu haben. Slotanus und sein junger Gehilfe arbeiteten offenbar recht kollegial mit den beiden herzoglichen Hofärzten, Dr. Johannes Weyer und Dr. Reiner Solenander, zusammen. Beide hatten in Italien die medizinische Ausbildung absolviert; von Solenander lernte Fabricius die Bedeutung gutdokumentierter Krankengeschichten als eines Erfahrungswertes, aus dem der Arzt/Chirurg in seinem Berufsleben so oft mit Gewinn schöpfen kann. Auch Weyer hatte einen nachhaltigen Einfluss auf des jungen Gehilfen Geisteswelt: Wie ein anderer berühmter Düsseldorfer, Friedrich von Spee, war der gute Doktor ein erbitterter Gegner des Hexenwahns, der Verfolgung, Folterung und juristisch untermauerten Ermordung Tausender unschuldiger Frauen und auch zahlreicher Männer.

Bald zog Fabricius auf Wanderschaft, bildete sich zunächst in Metz, dann in Genf bei Jean Griffon fort, der in Lausanne 1592 eine erfolgreiche Rhinoplastik durchgeführt hatte, fünf Jahre bevor Gaspare Tagliacozzi in Bologna diesen Eingriff beschrieb. Die Patientin war ein junges Mädchen, dem durchziehende Soldaten die Nase abgehackt hatten. Die neue, von Griffon aus einem Unterarmhautlappen geformte Nase heilte gut an; noch 19 Jahre nach dieser Pionieroperation begegnete Fabricius der Patientin in Genf und beobachtete entzückt, dass «die Markierungen von der Operation kaum sichtbar sind, nur im Winter verfärbt sich die Spitze der Nase gelegentlich etwas bläulich.»

\section{Fabricius wird die erste chirurgische}

\section{Entfernung von Gallensteinen}

\section{zugeschrieben}

Der Aufenthalt in Genf war noch in anderer Hinsicht von entscheidender Bedeutung für Fabricius. Er lernte die Buchdruckertochter Marie Colinet kennen und lieben und ehelichte die energische junge Frau am 30. Juli 1587. Sie wurde nicht nur die Mutter seiner acht Kinder (von denen nur eines, ein Sohn, den Vater überleben sollte), sondern auch eine Mitarbeiterin von aussergewöhnlichem Talent, eine Chirurgin, die Beinbrüche schiente, Patienten während des Gatten langen Abwesenheiten behandelte und, wie geschildert, als erste Heilkundige so weit bekannt überhaupt einen metallischen Fremdkörper mit einem Magneten aus der Hornhaut eines Auges entfernte. Die Pioniertat der Magnetextraktion wird in einem Akt der Ungerechtigkeit gegenüber Marie Colinet dem Chirurgen Hieronymus Brunschwig zugeschrieben, der schon 1497 Fremdkörper auf diese Art aus dem Auge holte jedoch wahrscheinlich «nur» aus dem Bindehautsack und nicht aus der schwieriger zu erreichenden Tiefe der Hornhaut. 
In Payerne, wo Fabricius 1602 Stadtchirurg wurde, lief nicht nur seine Praxis, sondern auch seine Publizistik auf Hochtouren. 1606 liess er in Basel die ersten hundert seiner Observationes oder Centuriae drucken, in schneller Reihenfolge kamen Abhandlungen über die Rote Ruhr, über Brandwunden und andere Herausforderungen an den Chirurgen hinzu. Die Berichte künden von teilweise kühnen Eingriffen wie der Beseitigung einer Analatresie, von traurigen Pflichten wie

\section{Die Berner machten ihn zum städtischen Amtschirurgen, eine Aufgabe, die er bis zu seinem Tod ausübte}

der Entfernung abgestorbener Föten aus dem Mutterleib und von einer Geissel der Medizin bis hinein ins 19. und 20. Jahrhundert, der Hilflosigkeit gegenüber Erkrankungen, die - wie wir heute wissen - auf Infektionen zurückzuführen waren. Dies belegt diese Geschichte aus seiner Zeit im Rheinland: «Ein 4 Jahre altes Mädchen, die Tochter des wohledlen Herrn N. von Hogstein zu den Volckerdehen, welches von einer akuten Krankheit genesen war, wurde von einem heftigen Fluss ergriffen, der zum Zahnfleisch hinzog und mit Übelkeit und Kopfschmerz einherging. Ihre Eltern führten dieselbe nach Düsseldorf, wo sie die hochberühmten Herren Dr. Galenus Weyer und Cosmas Slotanus (bei welchem ich damals tätig war) konsultierten. Das ganze Gesicht war ödematös geschwollen. An der linken Backe war eine runde Geschwulst entstanden von harter und blauer Beschaffenheit, jedoch nicht besonders gross. Das Zahnfleisch aber und die linke Seite des Mundes waren gangränös geworden. Wir haben viele Mittel, aber vergeblich angewandt, denn es war ein solch starker Zustrom von schleimiger und kalter Feuchtigkeit vorhanden, dass die eingepflanzte (natürliche) Wärme, welche durch die vorhergehende Krankheit schon geschwächt war, nicht mehr sich einstellen konnte. Und so ist denn die Kranke endlich, nachdem das Zahnfleisch und die Lippe bis hin zur Nase weggefressen waren, aus dem Leben geschieden.»

Fabricius war ein Generalist, operierte und behandelte fast alles, was von ihm verlangt wurde. In seiner 1607 in Basel erschienenen Schrift De Combustioni- bus fasste er die verschiedenen Methoden der Behandlung von Brandwunden zusammen und teilte sie als erster Autor in drei Schweregrade ein, mit dem ersten Grad als dem leichtesten und Brandwunden dritten Grades als den schwerwiegendsten. Fabricius wird ferner die erste chirurgische Entfernung von Gallensteinen, geschehen um 1618, zugeschrieben.

1615 zog Fabricius mit Marie nach Bern. Die Berner machten ihn zum städtischen Amtschirurgen, eine Aufgabe, die er bis zu seinem Tod ausübte - die für ihn geschaffene Stelle wurde nach seinem Ableben nicht wieder besetzt. Bis zuletzt arbeitete er nicht nur am Patienten, sondern auch an seinen zahlreichen Veröffentlichungen - wie an einem Werk, in dem er sich für die gewissenhafte Unterrichtung in jener Wissenschaft stark machte, die für ihn die Grundlage erfolgreicher chirurgischer Tätigkeit war: «Von der Fürtrefflichkeit und Nutz der Anatomy.» Am 15. Februar 1634 starb Wilhelm Fabricius von Hilden in Bern, betrauert von seiner Mitarbeiterin Marie. Der einzige überlebende Sohn Johann eilte, von des Vaters Krankheit hörend, aus der Ferne herbei, kam indes zu spät, da er auf dem Weg in die Schweiz nicht weniger als dreimal überfallen wurde - es waren wahrhaft unsichere Zeiten.

\section{Literatur zum Thema}

- Kompanje EJ. Painful sexual intercourse caused by a disproportionately long penis: an historical note on a remarkable treatment devised by Guilhelmus Fabricius Hildanus (1560-1634). Arch Sex Behav. 2006; 35:603-5.

- Jones E. The Life and Works of Guilhelmus Fabricius Hildanus (1560-1634). Med Hist. 1960; 4:112-134, 196-209.

- Kirkpatrick JJ, Curtis B, Fitzgerald AM, Naylor IL. A modern translation and interpretation of the treatise on burns of Fabricius Hildanus (1560-1634). Br J Plast Surg. 1995; 48:460-70.

- Rennekampf HO. Die Verbrennungsbehandlung in der Renaissance durch Fabricius Hildanus. Handchir Mikrochir Plast Chir. 2009; 41:355-358.

- Ruisinger MM. Misreading pictures: Fabricius Hildanus (1560-1634) and the cure of spinal dislocation. J Hist Neurosci. 2005; 14:334-40.

- Wennig W. Wilhelm Fabrys Lebenslauf. In: Jahrbuch der Universität Düsseldorf. Düsseldorf: Tritsch; 1971/72: 245-55. 